Editorial

\title{
Acknowledgment to Reviewers of Tomography in 2021
}

\section{Tomography Editorial Office}

Citation: Tomography Editorial Office. Acknowledgment to Reviewers of Tomography in 2021. Tomography 2022, 8, 281-283. https:// doi.org/10.3390/tomography8010022

Published: 29 January 2022

Publisher's Note: MDPI stays neutral with regard to jurisdictional claims in published maps and institutional affiliations.

Copyright: () 2022 by the author. Licensee MDPI, Basel, Switzerland. This article is an open access article distributed under the terms and conditions of the Creative Commons Attribution (CC BY) license (https://creativecommons.org/licenses/by/4.0/).

MDPI AG, St. Alban-Anlage 66, 4052 Basel, Switzerland

Rigorous peer-reviews are the basis of high-quality academic publishing. Thanks to the great efforts of our reviewers, Tomography was able to maintain its standards for the high quality of its published papers. Thanks to the contribution of our reviewers, in 2021, the median time to first decision was 25.5 days and the median time to publication was 55 days. The editors would like to extend their gratitude and recognition to the following reviewers for their precious time and dedication, regardless of whether the papers they reviewed were finally published:

Adam Trepczynski

Agnieszka Kotalczyk

Ahmed Othman

Akiko Todaka

Akram Abdo Almansoori

Alberto Chighine

Aleš Fidler

Alessandro N Franciosi

Alexander M. Long

Alexandra Roudenko

Alexandros G. Sykaras

Alfonso Vittorio Marchiano

Ali Al Kaissi

Alon Bartal

Amirmasoud Ahmadi

Ana Faustino

Andrea Alexandre

Andrea Borghesi

Andrea Cassoni

Andrea Gonzalez-Montoro

Andrew Lavender

Aneeza W. Hamizan

Angela Ammirabile

Anjul Khadria

Annalisa Carlucci

Antonio Tarasconi

Arash Navran

Aristides Hatjimihail

Arkady Serikov

Armando Cavallo

Arnaldo Stanzione

Attila Jakab

Barbara Spanò

Bhupal Ban
Bo Zhao

Bogdana Suchorska

Bojan V. Stimec

Bradford A Moffat

Caius Constantinescu

Carlos Figueiredo

Cengiz Akbulut

Cesare Gagliardo

Chad Quarles

Chengyue Wu

Chiranjibi Sitaula

Christian Booz

Christian Schach

Christopher P. Ober

Christos Michail

Claus Madsen

Clive Kelly

Cofano Fabio

Corinne Beinat

Corrado Angelini

Damiani Giovanni

Daniel Gónzalez-Bandala

Daniel McGowan

Daniela Messineo

Daniele Pironi

David Q.-H. Wang

Deborah Rund

Diana Sorina Feier

Dominic Gascho

Don Yoo

Donna E. Goldhawk

Dorota Oszutowska-Mazurek

Edvard Ehler

Edwin Dickinson 
Efstratios-Stylianos Pyrgelis

Ekaterina Tolstaya

Eleonora Cavallari

Ellen Funkhouser

Enrico Cassano

Etienne Rousseau

Eva Hassler

Federico Bolelli

Ferdinand Seith

Floris Van Velden

Francesca Arezzo

Francesco Coratti

Francesco D'Oria

Francesco Macri

Francesco Restelli

Francesco Verde

Frédéric Vella

Gaurav Sharma

Gautam Sethi

Georg Böning

Gerold Besser

Gilbert Lim

Gina Delia Roque-Torres

Giovanni Foti

Guglielmo Manenti

Gunnar Schuetz

Hamza Alizai

Héctor Espinós-Morató

Henrique Silva

Hipolito Nzwalo

Hiroaki Nakagawa

Hiroaki Toba

Hiroshi Kuroki

Hisham Bahmad

Hongming Shan

Hsieh-Fu Tsai

Hueisch Jy Ding

Hyung-youl Park

Indu G. Rajapaksha

Ines Fasolino

Ismini E Papageorgiou

Jan Mejzlik

Jasper Nijkamp

Jee Taek Kim

Jelena Prpić

Jerzy Narloch

Jia Ping Wu

Jinbin $\mathrm{Xu}$

Joana Damásio

John J. Sunderland

Jonathan Martinelli
Joost Van Der Vorst

Jung-Woo Lee

Jyh-Miin Lin

Karin Markenroth Bloch

Karl Olof Lövblad

Karolina Markiet

Ken-Ichiro Matsumoto

Kenneth Hettie

KeunBaDa Son

Ke-Vin Chang

Kevin Chen

Kirsten Koolstra

Kivovics Márton

Kiyotaka Nemoto

Klearchos Psychogios

Ko Hashimoto

Konrad P. Nesteruk

Kuo-Chih Chen

Lechuang Chen

Lina Padervinskienè

Lionel Fernel Gamarra

Lubomir Hadjiyski

Luca Burroni

Luca Giovanni Locatello

Luca Pasquini

Lu-Han Lai

Majid Jadidi

Malik Aydin

Manuel Durán-Poveda

Manuela Matesan

Marie-Luise Kromrey

Mark J. Roef

Martijn Cloos

Martina C. Fuss

Mary-Kate Hayward

Masaki Fukunaga

Massimo Bonacchi

Mateusz Krystian Hołda

Matteo Fermi

Matthew F. Covington

Mattia Chiesa

Maxime Ronot

Michael Garwood

Michal Studniarek

Michela Gabelloni

Miguel Alcaraz

Mihaela Zegrean

Mikalai Budzevich

Milagros Hidalgo De La Cruz

Mingchung Chou

Mirjam Gerwing 
Mohamed Elsharkawy

Mohammed R. S. Sunoqrot

Mohd Parvez Khan

Mónica Martins

Mostafa Kamal Masud

Mugurel Constantin Rusu

Mukli Péter

Munish Chauhan

Naoki Ohno

Nassr Al-Nuaimi

Natalia D. Gladkova

Nektarios Koukourakis

Nguyen Quoc Khanh Le

NIcholas Sisco

Niels Qvist

Oganes Ashikyan

Oke Gerke

Paola Suárez-Meade

Paolo Campioni

Paolo Prandoni

Paolo Spinnato

Patrycja Sosnowska-Sienkiewicz

Paul E. Sijens

Paul Monsarrat

Paul Schoenhagen

Paul-Andrei Stefan

Paulo J. Palma

Peiman Habibollahi

Petros Christopoulos

Pier Luigi Stefàno

Piergiorgio Solli
Piero Vincenzo Lippolis

Piotr Leszczyński

Piotr Morasiewicz

Rahmeh Othman

Rajikha Raja

Ralph A. Bundschuh

Reza Piri

Robert Cristian Purdoiu

Robert Nordstrom

Roddy Hiram

Sadiya Shaikh

Sebastian Nagel

Selena Milicevic Sephton

Sergey Pavlovich Osipov

Sergio Salerno

Seungryong Cho

Shiho Naito

Shuiyu Lu

Siddharth Thaker

Sneha Raju

Sophie Richter

Špela Šalamon

Subhamoy Mandal

Susanne Schnell

Sven Månsson

Tetsuya Ishikawa

Tolga Özmen

Vasileios Siokas

Yeou-Jiunn Chen

Yong-An Chung

Yuki Hashimoto 